\title{
Secretion of Cholesterol-Rich Lipoproteins by Perfused Livers of Hypercholesterolemic Rats
}

\author{
Simon-Pierre Noel, Laurence Wong, Peter J. Dolphin, Ladislav Dory, \\ and DAvid RuBINSTEIN, Department of Biochemistry, McGill University, \\ Montreal, Quebec, Canada H3G 1 Y6
}

A B S T RACT Rats maintained on a high-fat diet supplemented with propylthiouracil develop a hypercholesterolemia, an increased serum level of apolipoprotein (apo) E, abnormal very low density lipoproteins (VLDL) and low density lipoproteins (LDL), and a fatty liver which contains cholesterol ester as its major lipid. The fatty liver secretes apoE into a recirculating perfusate at a significantly higher rate and produces cholesterol ester-rich, apoC-deficient VLDL with slower electrophoretic mobility than the triacylglycerol-rich VLDL produced by perfused normal livers. LDL, secreted in significant quantities by the perfused fatty liver, but not by the normal liver, is also cholesterol rich and contains apoE as well as apoB. The incorporation of $\left[{ }^{3} \mathrm{H}\right]$ leucine into apoVLDL and apoLDL secreted by the livers of the hypercholesterolemic animals and the apoVLDL secreted by the normal liver corresponds to the pattern visualized when the apoproteins are separated by polyacrylamide gel electrophoresis. Similar patterns are noted when nonrecirculating perfusates are studied. These results indicate that the cholesterol ester-rich, apoC-deficient VLDL and the apoE-containing LDL found in the serum of hypercholesterolemic rats are not solely catabolic remnants of VLDL and chylomicrons but are secreted by the liver. Separation of the perfusate lipoproteins by agarose gel filtration revealed that

\footnotetext{
A portion of this work was presented to the American Heart Association, November 1978, Dallas, Tex., and appeared in abstract form in 1978. Circulation. 58: II-77.

Dr. Wong is a Fellow of the Canadian Heart Foundation. Dr. Dolphin is a Scholar of the Canadian Heart Foundation. Dr. Noel's present address is Département de Biochimie, Université de Montréal, Montréal, Quebec, Canada; Dr. Wong's present address is The Rockefeller University, New York; Dr. Rubinstein and Dr. Dolphin's present address is Department of Biochemistry, Dalhousie University, Halifax, Nova Scotia, Canada; and Dr. Dory's present address is Department of Physiology, Louisiana State University, New Orleans, La. Address reprint requests to Dr. Rubinstein.

Received for publication 7 August 1978 and in revised form 21 March 1979.
}

most of the apoE secreted by the livers of hypercholesterolemic rats is found in the VLDL and LDL, whereas apoE secreted by the normal livers is distributed equally between VLDL, high density lipoproteins, and a low molecular weight fraction which corresponds to the virtually delipidated apoprotein. Thus the distribution of apoE among the lipoprotein fractions may be related to the total amount of cholesterol being transported in the circulation.

\section{INTRODUCTION}

Primary familial dysbetalipoproteinemia (Type III hyperlipoproteinemia, broad-beta disease) is characterized by the presence of very low density lipoproteins $(\mathrm{VLDL})^{1}$ showing $\beta$-mobility on electrophoresis, containing abnormally high amounts of both cholesterol and apolipoprotein (apo) E (arginine-rich protein), and a decreased proportion of apoC $(1,2)$. Based on these properties and a number of metabolic studies, it has been suggested that the abnormal VLDL found in familial dysbetalipoproteinemia represents an accumulation of remnants of VLDL and chylomicron catabolism which have not been efficiently removed $(2-6)$.

The presence of similar $\beta$-migrating VLDL (BVLDL) having a high cholesterol and apoE content and little or no apoC can be detected in the sera of a variety of animal species fed a high-cholesterol diet, especially if hypothyroidism is induced simultaneously. A lipoprotein fraction having a hydrated density in the low density lipoprotein (LDL) range with an apoprotein composition-resembling high density lipoprotein (HDL) $\left(\mathrm{HDL}_{\mathrm{c}}\right)$ is also found $(7-12)$. It has been suggested that, like the abnormal lipoproteins of human dysbetalipoproteinemia, the $\beta$-migrating lipoproteins

${ }^{1}$ Abbreviations used in this paper: apo, apolipoprotein(s); B-VLDL, $\beta$-migrating very low density lipoprotein(s); HDL, high density lipoprotein(s); LDL, low density lipoprotein(s); VLDL, very low density lipoprotein(s). 
found in the hypercholesterolemic serum of animals represent VLDL remnants $(8,13)$, although the amount of apoE in these lipoproteins may be too great to be derived solely from the catabolism of VLDL (9).

We have recently observed that VLDL isolated from perfusates of livers taken from hypercholesterolemic rats exhibit some of the immunological properties of the abnormal circulating VLDL, including a relative deficiency of apoC (14). This observation may make it possible to determine directly whether the abnormal VLDL and LDL found in the serum of hypercholesterolemic rats are synthesized and secreted by the liver. The results to be reported here indicate that at least a portion of the abnormal lipoproteins found in the hypercholesterolemic rat serum are secreted directly by the liver.

\section{METHODS}

Experimental animals. Male hooded rats weighing 300-350 g were maintained on a Purina Chow (Ralston Purina Co., St. Louis, Mo.) or a high-fat diet which included $40 \%$ butter fat, $5 \%$ cholesterol, $0.2 \%$ choline chloride, $0.1 \%$ sodium cholate, and $0.3 \%$ propylthiouracil (Thrombogenic diet; ICN Nutritional Biochemicals, Cleveland, Ohio). Rats placed on the high-fat diet for periods of up to $6 \mathrm{wk}$ maintained but did not gain weight. These animals developed grossly fatty livers. The rats were anaesthetised with Nembutal (Abbott Laboratories, North Chicago, Ill.) before cannulation of the portal vein. For the studies of the effect of diets on serum lipids, blood samples were obtained by cardiac puncture.

Liver perfusions. Livers from normal and hypercholesterolemic rats were perfused by a modification (15) of the technique of Miller et al. (16) using a silastic tubing lung (17). The perfusate consisted of Krebs-Ringer bicarbonate buffer, pH 7.4, which contained $0.1 \%$ glucose. Fresh human erythrocytes, washed four times, were suspended in the perfusate to give a hematocrit of $25 \%$. Replacement of human erythrocytes by rat erythrocytes did not alter the rate of secretion of the lipoproteins. The liver was flushed with $\cong 30 \mathrm{ml}$ of perfusate in situ immediately after cannulation, then transferred to the perfusion apparatus after which $100 \mu \mathrm{Ci}$ $\left[4,5-{ }^{3} \mathrm{H}\right]$ leucine (New England Nuclear, Montreal, Quebec) was added to $40 \mathrm{ml}$ of perfusate recirculating at a rate of $\cong 1 \mathrm{ml} / \mathrm{g}$ liver per min. A perfusate of similar composition was used at a flow rate of $10 \mathrm{ml} / \mathrm{min}$ during 2 -h nonrecirculating perfusions. Bile flow averaged $40 \mu \mathrm{l} / \mathrm{g}$ liver per $\mathrm{h}$ from the control liver and $9 \mu \mathrm{l} / \mathrm{g}$ per $\mathrm{h}$ from the fatty liver. Despite this difference in bile flow, the total cholesterol secreted in the bile by the two types of livers was not very different $(2.8$ and $2.4 \mu \mathrm{g} / \mathrm{g}$ liver per $h$ for control and hypercholesterolemic livers, respectively) as a result of the higher concentration of cholesterol in the bile of the fatty livers.

Analysis of lipoproteins. Lipoproteins were isolated from the liver perfusates or serum by ultracentrifugation in a Beckman L5-50 preparative ultracentrifuge (Beckman Instruments, Inc., Spinco Div., Palo Alto, Calif.) with an SW 41 rotor using a modification of the method of Havel et al. (18). Sequential addition of solid $\mathrm{NaBr}$ (19) was used to obtain the following densities: VLDL, <1.006 $\mathrm{g} / \mathrm{ml}$; LDL, 1.006$1.087 \mathrm{~g} / \mathrm{ml}$; HDL, $1.087-1.21 \mathrm{~g} / \mathrm{ml}$. Each lipoprotein fraction was washed once with a solution of the appropriate density which contained $0.01 \%$ EDTA, and then dialyzed against saline that contained $0.01 \%$ EDTA and $0.02 \% \mathrm{NaN}_{3}$. The electrophoretic mobilities of the isolated lipoproteins were determined by a modification of the procedure of Noble (20) using a $0.8 \%$ agarose gel which contained $0.5 \%$ bovine serum albumin (21). Perfusate lipoproteins were also separated on a $2.5 \times 140-\mathrm{cm}$ Bio-Gel A-0.5m agarose (Bio-Rad Laboratories, Richmond, Calif.) column (22) by elution with a $0.9 \% \mathrm{NaCl}, 0.01 \%$ EDTA, $0.02 \% \mathrm{NaN}_{3}$ buffer ( $\mathrm{pH} \mathrm{7.3)} \mathrm{at}$ a rate of $20 \mathrm{ml} / \mathrm{h}$. Each column was recalibrated with isolated lipoproteins, and the elution pattern was checked with rat serum. $90-110 \%$ of the applied apoE was recovered in the column eluates. When isolated serum VLDL was applied to the column, apoE was recovered in the void volume, with only negligible quantities appearing in the eluates from the remainder of the column. The perfusates were concentrated threefold by dialysis against Aquacide 11-A (CalbiochemBehring Corp., American Hoechst Corp, Calif. ) before being applied to the column. The concentration procedure had no effect on the distribution of the lipoproteins or their apoproteins.

Isolated lipoproteins were delipidated twice in ethanolether 3:1 (23) at $-10^{\circ} \mathrm{C}$ and solubilized in Tris-glycine buffer, pH 8.9, which contained $7 \mathrm{M}$ urea and $1 \%$ solium dodecyl sulfate. The apoproteins were separated by electrophoresis on polyacrylamide gel for 4-5 h (24), and the gel bands were identified by comparison with the migration of purified rat apoproteins (14). In some experiments the electrophoresis was run for $25-30 \mathrm{~h}$ in $250-\mathrm{mm}$ tubes to further separate closely migrating bands. Radioactivity associated with apoproteins was counted after oxidation of the stained gel bands (25).

Analytical procedures. ApoE was determined by a modification (12) of the immunoelectrophoretic procedure of Laurell (26) in which 0.1\% Triton X-100 (Rohm and Haas Co., Philadelphia, $\mathrm{Pa}$.) and $1 \%$ bovine serum albumin were added to each aliquot being assayed. Triton X-100 was also added to the gel and the buffers. The interassay and intraassay variations were $<3 \%$ at all concentrations when carried out within $3 \mathrm{~d}$. The electroimmunoassay was validated by delipidating rat VLDL and dissolving the apoproteins in $4 \mathrm{M}$ guanidine hydrochloride. The apoproteins were then separated on a BioGel A 1.5-M column (12), and the protein content of the purified apoE was measured directly by dye binding (27). These measurements agreed within $10 \%$ with the apoE level obtained by direct electroimmunoassay of the intact VLDL.

Hepatic and serum lipids were extracted by the method of Folch et al. (28), and the isolated perfusate lipoproteins were extracted by the ethanol-ether mixture used for delipidation. The lipids were separated on thin-layer chromatography with silica gel $\mathrm{G}$ and a solvent system of petroleum ether, diethyl ether, and acetic acid, 90:10:1 (vol/vol). The phospholipids were eluted from the silicic acid plates with chloroform-methanol 2:1 and the neutral lipids with chloroform. Phospholipids were digested with perchloric acid, and the liberated inorganic phosphate was measured by the method of Fiske and SubbaRow (29). Triacylglycerols were determined according to Van Handel (30) and cholesterol by the method of Zlatkis et al. (31). Perfusate triacylglycerol lipase was assayed by the procedure of Nilsson-Ehle and Schotz (32). Protein was measured by the method of Lowry et al. (33). DNA was extracted from $1 \mathrm{~g}$ of liver and determined by the diphenylamine reaction $(34,35)$.

\section{RESULTS}

Rats maintained for $6 \mathrm{wk}$ on the high-fat diet employed in these experiments developed a hyperlipemia, especially hypercholesterolemia, and an increase in serum level of apoE $(12,36)$. Before commencing liver 
perfusions, the degree and persistence of these changes in sera of postabsorptive (7-h-fasted) animals were determined. The data are shown in Fig. 1. The cholesterol ester level rose rapidly during the first $20 \mathrm{~d}$ of the high-fat diet, then stabilized, whereas free cholesterol, although somewhat elevated, did not rise further until about the $30 \mathrm{~d}$. The level of apoE rapidly became elevated and continued to increase during the ensuing period. Upon restoration of the normal diet the free and esterified cholesterol levels fell, but that of apoE remained high for at least $1 \mathrm{wk}$. Electrophoresis of the serum lipoproteins on agarose gels revealed the presence of B-VLDL $6 \mathrm{~d}$ after the high-fat diet had been replaced by Purina Chow (similar to line 4 in Fig. 3).

The liver accumulated large quantities of cholesterol esters during the first $4 \mathrm{wk}$ of the hyperlipemiainducing diet. The cholesterol ester level then remained elevated, even after cessation of the highfat diet. Smaller increments of free cholesterol and triacylglycerols, and a transient increase in phospholipids was also observed (not shown in Fig. 1). The persistence of the cholesterol ester-loaded fatty liver, B-VLDL, and the elevated serum level of apoE, suggest that livers from hypercholesterolemic rats could reflect their in vivo status when perfused in vitro with an artificial lipid-free medium.

The pattern of secretion of apoE by perfused livers from normal and hypercholesterolemic animals is shown in Fig. 2. The secretion of apoE by the control livers into the perfusate is linear throughout the 4-h perfusion, which confirms recent observations of Felker et al. (37). In contrast, there is a rapid initial release of apoE by the livers from the hypercholesterolemic animals, followed by a linear secretion. To determine to what extent the initial release of apoE by livers from the hypercholesterolemic rats represents lipoproteins in contaminating serum or extracellular fluid in equilibrium with the circulation, $\left[{ }^{14} \mathrm{C}\right]$ dextran (mean 70,000 mol wt, New England Nuclear) was administered intraveneously to hypercholesterolemic animals. The livers were cannulated for perfusion $15 \mathrm{~min}$ later, and the ratio of ${ }^{14} \mathrm{C}$ to apoE in the serum just before cannulation was compared with that found in the liver perfusate after $15 \mathrm{~min}$ of recirculation. The results (not shown) indicate that a maximum of one-third of the apoE released during the first $15 \mathrm{~min}$ of perfusion could be accounted for by lipoproteins of the serum and equilibrated extracellular fluid.

The rate of secretion of apoE by the perfused livers was derived by least squares analysis of the linear portion of the time-course in individual experiments. The cumulative data is presented in Table I. It will be noted that the rate of secretion of apoE by the perfused livers from the hypercholesterolemic rats was significantly greater than that of the controls, whether expressed as micrograms per gram wet weight of liver per hour, or micrograms per milligram DNA per hour. The latter compensates for any distortion of secretion

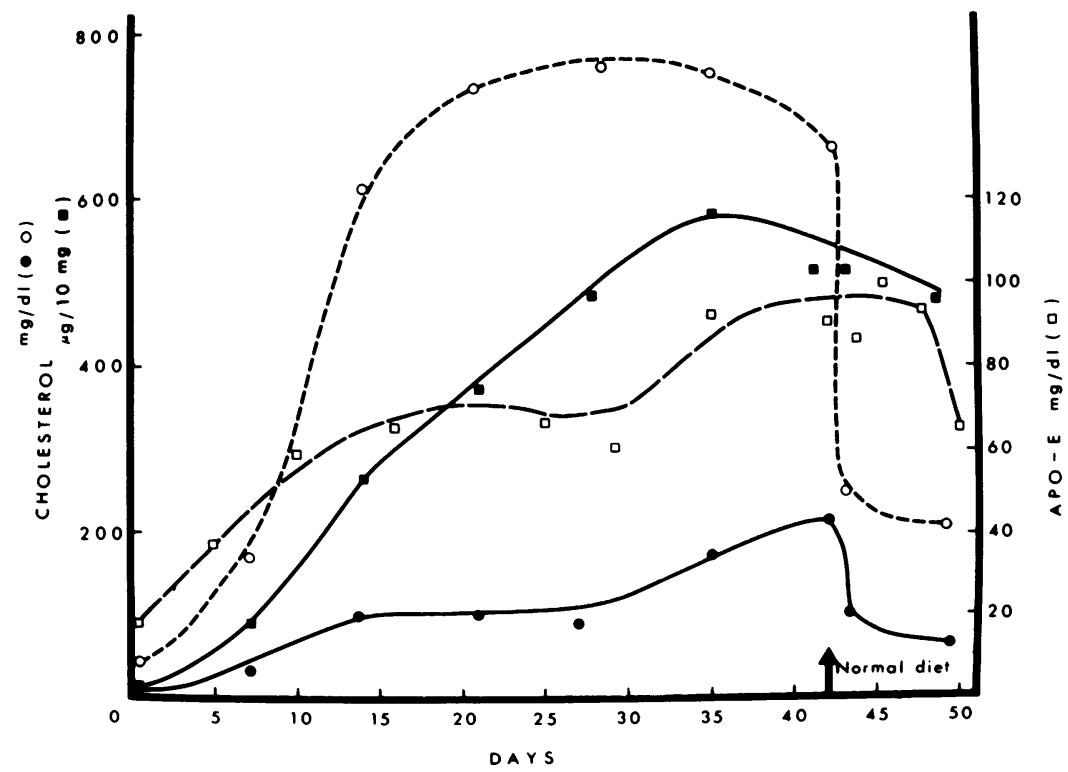

FIGURE 1 Changes in levels of serum apoE, free and esterified cholesterol and hepatic cholesterol esters during the high-fat diet. The animals were allowed to feed ad libitum until $7 \mathrm{~h}$ before sacrifice when food was withdrawn. After $42 \mathrm{~d}$, a normal chow diet was substituted for the high-fat diet. Each point is the mean of two animals which gave results within $10 \%$ of each other. (O) serum cholesterol esters; (O) serum free cholesterol; ( $\square$ ) serum apoE; ( $\square$ ) liver cholesterol esters. 


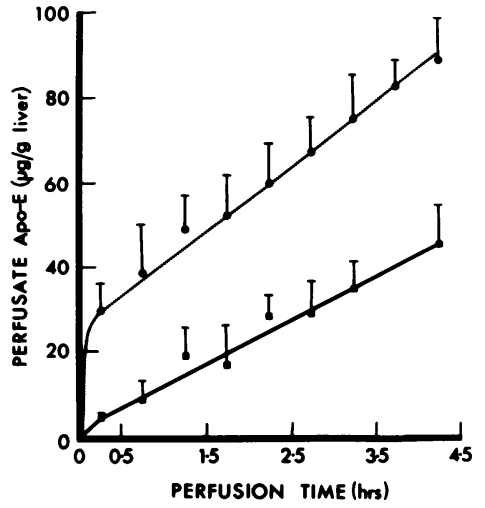

Figure 2 The secretion of apoE by perfused livers from control ( $\square)$ and hypercholesterolemic $(\bullet)$ rats. Each point represents the mean and SEM of at least 10 experiments.

rates introduced by the fatty state of the livers of the hypercholesteolemic animals (Fig. 1 and Table II). The initial release of preformed lipoproteins by the perfused livers, calculated from the intercepts of the linear portions of the slopes illustrated in Fig. 2, is shown in Table I. In the perfusates of livers from the hypercholesterolemic rats the initial release represents $\cong 30 \%$ of the total apoE collected in $4 \mathrm{~h}$, but is negligible in perfusates of normal livers. To restrict the studies to lipoproteins actively secreted by the livers, the perfusates were routinely replaced after the first 15 min in all subsequent experiments, resulting in initial releases which were close to zero. However, the significant difference between the rates of secretion of apoE by livers from control and hypercholesterolemic rats persisted. In some experiments, the lipid composition and electrophoretic pattern of the apoproteins of VLDL released during the first 15 min were compared with those seen after the additional 4-h perfusion. No significant differences could be detected (data not shown).

The electrophoretic mobility in agarose gel of the isolated perfusate lipoproteins is shown in Fig. 3. The migration of the VLDL secreted by the liver from hypercholesterolemic rats is retarded compared with that secreted by the normal liver, although it still has pre$\beta$-mobility. The liver from the hypercholesterolemic rat also secretes LDL of $\beta$-mobility which is virtually absent in control perfusates. No evidence could be found for the presence of cathodal-migrating Lp-X in the perfusates when the total lipoproteins or isolated LDL were submitted to electrophoresis in agar gels (38).

The protein and lipid content of the ultracentrifugally isolated lipoproteins secreted by the perfused livers is shown in Table II. Most of the apoprotein secreted by the livers from normolipemic rats is found in the VLDL, with virtually none in LDL. Although the total quantity of apoprotein found in the perfusates of livers from hypercholesterolemic rats is not significantly greater, it is distributed more evenly between the VLDL and LDL. The experimental livers secrete considerably more lipid, primarily in LDL. It should also be noted that livers from the hypercholesterolemic animals contain $\cong 10$ times as much lipid as the normal livers. This correlates with the grossly fatty appearance of the experimental livers.

The lipid composition of the lipoproteins secreted by the perfused livers is shown in Table III. While VLDL from the control animals contains primarily triacylglycerols, cholesterol ester is the predominant lipid in both the VLDL and LDL secreted by the livers of hypercholesterolemic rats, apparently reflecting the latter's lipid content. Thus the cholesterol esterrich fatty liver secretes abnormal VLDL, in which

TABLE I

Rate of Secretion of ApoE by Perfused Livers

\begin{tabular}{|c|c|c|c|}
\hline & Control & Hypercholesterolemic & $P$ \\
\hline \multicolumn{4}{|l|}{$\begin{array}{l}\text { Perfusate collection period } \\
\qquad(0-4 \mathrm{~h})\end{array}$} \\
\hline Secretion, $\mu g / g$ liver/h & $10.6 \pm 1.1(11)$ & $14.4 \pm 1.4(12)$ & $<0.005$ \\
\hline Secretion, $\mu g / m g ~ D N A / h$ & $4.2 \pm 0.3(5)$ & $5.6 \pm 0.5(5)$ & $<0.05$ \\
\hline Initial release, ${ }^{*} \mu g / g$ liver & $4.1 \pm 1.3$ & $28.1 \pm 6.2$ & \\
\hline \multicolumn{4}{|l|}{$\begin{array}{l}\text { Perfusate collection period } \downarrow \\
\quad(15 \mathrm{~min}-4 \mathrm{~h}, 15 \mathrm{~min})\end{array}$} \\
\hline Secretion, $\mu g / g$ liver/h & $11.8 \pm 0.7(5)$ & $17.8 \pm 1.3(5)$ & $<0.05$ \\
\hline Initial release, ${ }^{*} \mu g / g$ liver & $0.2 \pm 0.2$ & $6.8 \pm 3.2$ & \\
\hline
\end{tabular}

Each figure is the mean of the number of experiments indicated in parenthesis \pm SEM.

* Calculated from the intercept of the linear portion of the time-course during perfusion (Fig. 2).

After 15 min of perfusion, the perfusate was discarded, and fresh perfusate was recirculated for an additional $4 \mathrm{~h}$. 
TABLE II

Protein and Lipid Content of Lipoproteins Secreted by Perfused Livers

\begin{tabular}{|c|c|c|c|c|}
\hline & \multicolumn{2}{|c|}{ Protein } & \multicolumn{2}{|c|}{ Lipid } \\
\hline & Control & Hypercholesterolemic & Control & Hypercholesterolemic \\
\hline VLDL, $\mu g / g$ liver $4 h$ & $95 \pm 6$ & $51 \pm 10$ & $420 \pm 73$ & $380 \pm 90$ \\
\hline LDL, $\mu g / g$ liver $4 h$ & $2 \pm 1$ & $49 \pm 13$ & $47 \pm 26$ & $273 \pm 66$ \\
\hline HDL, $\mu g / g$ liver $/ 4 h$ & $11 \pm 6$ & $30 \pm 6$ & $13 \pm 6$ & $46 \pm 6$ \\
\hline Liver, $m g / g$ liver & & & $8.5 \pm 0.5$ & $84.9 \pm 7$ \\
\hline
\end{tabular}

Values represent the mean $\pm S E M$ of three and five perfusion of livers from control and hypercholesterolemic animals, respectively, and are based on the wet weight of the livers.

cholesterol esters replace triacylglycerols as the dominant lipid, and cholesterol-rich LDL, a lipoprotein which is virtually absent from the perfusates of normal livers.

A comparison of the apoprotein composition of the VLDL and LDL secreted by perfused livers from normolipemic and hypercholesterolemic animals, as visualised by polyacrylamide gel electrophoresis, is shown in Fig. 4. The VLDL secreted by the livers of hypercholesterolemic animals contains two distinct bands in the apoE region of the gel which can be readily separated by the use of longer gels (Fig. 4B). The two bands, eluted separately by electrodialysis (39), reacted with goat anti-rat apoE serum on immunodiffusion. Neither band reacted with anti-rat albumin, anti-apoA-1, anti-apoB, or anti-apoC, nor did their mobility correspond to apoA-1 when separated by isoelectric focussing. These observations do not rule

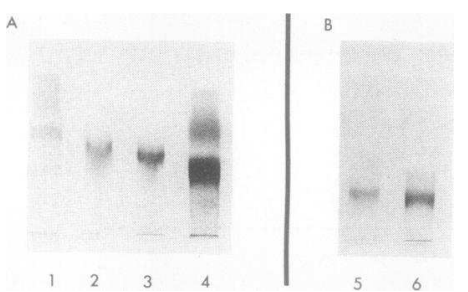

FIGURE 3 Electrophoresis in agarose of serum and perfusate lipoproteins. (1) Normal rat serum; (2) VLDL secreted by perfused liver from normal animals; (3) VLDL secreted by perfused liver from hypercholesterolemic rats; (4) serum from hypercholesterolemic rats; (5) LDL secreted by perfused liver from hypercholesterolemic rats; (6) LDL isolated from the serum of hypercholesterolemic rats. Gels depicted in $3 \mathrm{~A}$ and $3 B$ were run at different times so that the mobility of the lipoproteins on the two gels are not directly comparable. Lane 1 contains undiluted serum, lane 4 serum diluted 1:1 with saline. Lanes 3 and 4 contain equal amounts of isolated VLDL (as determined by nephelometry). Lane 5 has $\cong 50 \%$ of the LDL of lane 6 . The bands in lane 1 , from the origin are LDL, VLDL, and HDL, as determined by isolated lipoproteins. The somewhat decreased mobility of normal liver perfusate VLDL when compared with serum VLDL has previously been reported (48). out the possibility that the lower band contains an as yet unidentified additional apoprotein(s). However, the separation of rat apoE into two bands by polyacrylamide gel electrophoresis in urea has previously been noted (40). In contrast to the normal VLDL, that secreted by rat livers taken from hypercholesterolemic animals appears to be almost devoid of apoC. Whereas LDL secreted by both types of livers apparently contain apoB at the top of the running gel, LDL from livers of hypercholesterolemic animals also contain the double band in the apoE region.

To determine if the abnormal VLDL and LDL secreted by livers from hypercholesterolemic rats were synthesized de novo, $\left[{ }^{3} \mathrm{H}\right]$ leucine was added to the perfusate. The distribution of the radioactive precursor among the major apoproteins of VLDL secreted by the two types of liver, shown in Table IV, reflects the differences in the apoprotein patterns after separation on polyacrylamide gel electrophoresis (Fig. 4). The relative incorporation of $\left[{ }^{3} \mathrm{H}\right]$ leucine into apoB and the upper band of apoE was similar, but the VLDL secreted by livers from hypercholesterolemic animals showed a markedly increased percentage of radioactivity in the lower apoE band, whereas incorporation into the apoC bands was very much diminished. Similarly LDL secreted by the livers from the hypercholesterolemic animals showed $\left[{ }^{3} \mathrm{H}\right]$ leucine incorporation in apoB and in the two bands of the apoE region, the lower having the greater radioactivity. The negligible incorporation into the rapidly migrating apoC bands of the LDL confirms their virtual absence from the polyacrylamide gels (Fig. 4).

Although these data indicate that livers from the hypercholesterolemic rats directly. secrete abnormal lipoproteins into the perfusate, their possible formation during recirculation through the fatty liver was considered. Therefore, livers from normal and hypercholesterolemic rats were perfused in a nonrecirculating system. Typical experiments, shown in Table $\mathrm{V}$, indicate that, as is the case in the recirculating system, the normal liver largely secretes triacylglycerolrich VLDL. The small amount of material found in the 
TABLE III

Lipid Composition of Livers and of Lipoproteins Secreted by Perfused Livers

\begin{tabular}{|c|c|c|c|c|c|c|c|c|}
\hline & \multicolumn{4}{|c|}{ Control } & \multicolumn{4}{|c|}{ Hypercholesterolemic } \\
\hline & TG & $\mathrm{CE}$ & FC & PL & TG & CE & FC & PL \\
\hline & \multicolumn{8}{|c|}{$\%$ distribution } \\
\hline VLDL & $71 \pm 2$ & $7 \pm 2$ & $7 \pm 1$ & $16 \pm 2$ & $35 \pm 2$ & $42 \pm 3$ & $11 \pm 1$ & $12 \pm 1$ \\
\hline LDL & $39 \pm 4$ & $19 \pm 7$ & $18 \pm 5$ & $25 \pm 4$ & $12 \pm 2$ & $49 \pm 7$ & $19 \pm 2$ & $21 \pm 5$ \\
\hline HDL & $14 \pm 1$ & $42 \pm 1$ & $16 \pm 4$ & $28 \pm 5$ & $9 \pm 2$ & $34 \pm 3$ & $27 \pm 4$ & $30 \pm 3$ \\
\hline Liver & $31 \pm 2$ & $3 \pm 0.5$ & $8 \pm 1$ & $58 \pm 3$ & $13 \pm 3$ & $79 \pm 2$ & $3 \pm 1$ & $6 \pm 2$ \\
\hline
\end{tabular}

Each value is the mean \pm SEM of three and six experiments with livers from control and hypercholesterolemic animals, respectively. It should be noted that the lipid composition of the LDL secreted by the control liver and the HDL secreted by both the control and hypercholesterolemic livers represent relatively small quantities when compared with the other lipoprotein fractions (Table II) but are provided for completeness. Abbreviations used in this table are: TG, triacylglycerols; CE, cholesterol esters; FC, free cholesterol; PL, phospholipids.

LDL density range appears to be characteristic of the nonrecirculating system, for it has previously been observed by Marsh (41) who demonstrated that it is not serum LDL (42). Both VLDL and LDL secreted into the nonrecirculating perfusate by the liver from the hypercholesterolemic rats are cholesterol-ester rich, the LDL containing about one-half of the total apoprotein. The lipoproteins were delipidated and their apoproteins submitted to polyacrylamide gel electrophoresis. The resulting gel patterns and dis-
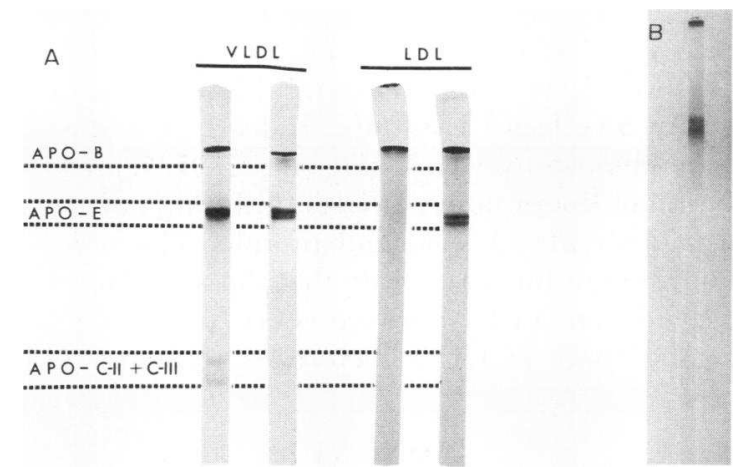

Figure 4 Polyacrylamide gel electrophoretic patterns of apoproteins secreted by perfused livers. The gels were loaded with $50 \mu \mathrm{g}$ of apoprotein, except for apoLDL from the normal liver perfusates where only $15 \mu \mathrm{g}$ was available. In subsequent experiments, $50 \mu \mathrm{g}$ of pooled apoLDL from normal liver perfusates was applied, but no apoE bands were visualized. Identity of the apoprotein bands was established by comparison with the mobility of purified apoproteins. (A) Regular electrophoresis gels. (B) apoVLDL secreted by perfused liver from a hypercholesterolemic rat separated by electrophoresis on long $(250 \mathrm{~mm})$ gels. Note the clear separation of the upper and lower bands in the apoE region. (N) lipoprotein secreted by livers taken from normal animals; (HC) lipoproteins secreted by livers taken from hypercholesterolemic rats. tribution of incorporated $\left[{ }^{3} \mathrm{H}\right]$ leucine (not shown) were similar to those shown in Fig. 4 and Table IV, with prominent, rapidly migrating apoC bands in the normal VLDL but only traces in the VLDL secreted by the hypercholesterolemic livers. Whereas the LDL from the hypercholesterolemic liver contained significant quantities of apoE, that secreted by the normal contained no visible apoE (although small amounts could be detected by electroimmunoassay). Thus the abnormal lipoproteins found in the perfusates of the livers of the hypercholesterolemic animals are not formed from normal lipoproteins during recirculation through the fatty liver.

The possibility that cholesterol ester-rich VLDL or LDL was formed by the action of a triacylglycerol lipase released into the perfusate by livers from hypercholesterolemic rats was also considered. Although a lipase was detected in both normal and experimental liver perfusates, its activity was far too low to account for the formation of the cholesterol ester-rich VLDL

TABLE IV

Distribution of $\left[{ }^{3} \mathrm{H}\right]$ Leucine Incorporated into the Apo Secreted by the Perfused Livers

\begin{tabular}{lrccc}
\hline & \multicolumn{2}{c}{ Control } & & \multicolumn{2}{c}{ Hypercholesterolemic } \\
\cline { 5 - 5 } \cline { 5 - 5 } \multicolumn{1}{c}{ Apoprotein } & VLDL & & VLDL & LDL \\
\hline & & \% distribution \pm SEM \\
ApoB & $23 \pm 4$ & $27 \pm 1$ & $50 \pm 3$ \\
ApoE (upper band) & $21 \pm 5$ & $19 \pm 2$ & $11 \pm 1$ \\
ApoE (lower band) & $9 \pm 1$ & $28 \pm 4$ & $14 \pm 1$ \\
ApoC (II and III) & $28 \pm 4$ & $6 \pm 2$ & $1 \pm 0.2$ \\
\hline
\end{tabular}

Each figure is the mean of four perfusions each of 4-h duration. Radioactivities apply to the gels ranged from 2,000 to $40,000 \mathrm{cpm}$, with recoveries of $70-110 \%$. 
TABLE V

Secretion of Lipoproteins into a Nonrecirculating Perfusate

\begin{tabular}{|c|c|c|c|c|}
\hline & \multicolumn{2}{|c|}{ Normal } & \multicolumn{2}{|c|}{$\begin{array}{l}\text { Hypercho- } \\
\text { lesterolemic }\end{array}$} \\
\hline & VLDL & LDL & VLDL & LDL \\
\hline \multicolumn{3}{|c|}{ ApoE secretion, $\mu g / g / h \ldots \ldots$} & \multicolumn{2}{|c|}{27} \\
\hline \multicolumn{5}{|l|}{ Lipoprotein secretion } \\
\hline Protein, $\mu g / g / h$ & 29 & 5 & 13 & 17 \\
\hline Lipid, $\mu g / g / h$ & 96 & 19 & 140 & 148 \\
\hline \multicolumn{5}{|l|}{ Distribution } \\
\hline Triacylglycerols, \% & 61 & 27 & 20 & 5 \\
\hline Cholesterol, \% & 26 & 53 & 63 & 52 \\
\hline Phospholipid, \% & 13 & 20 & 17 & 43 \\
\hline
\end{tabular}

The data are representative of two experiments with each type of liver. Both experiments gave similar patterns. The livers were perfused for $2 \mathrm{~h}$, after discarding the perfusate used during the initial $15 \mathrm{~min}$. The collected perfusate lipoproteins were then isolated. In one set of experiments the livers were perfused for only $1 \mathrm{~h}$ before the lipoproteins were isolated. A pattern similar to that shown above was obtained.

and LDL from normal VLDL. However, the detection of slight perfusate lipase activity makes it impossible to rule out the formation of some cholesterol ester-rich LDL from the corresponding VLDL, which already is triacylglycerol poor. Nevertheless, cholesterol ester-rich LDL, which have an apoprotein electrophoresis pattern similar to LDL secreted by the fatty livers (Fig. 4) can be isolated from hepatic Golgi apparatus of the hypercholesterolemic rats. ${ }^{2}$

The distribution of electroimmunoassayable apoE among the various ultracentrifugally isolated perfusate lipoproteins is shown in Table VI. In contrast with the normal perfused livers, those from the hypercholesterolemic rats secreted a significant portion of the apoE in the $d: 1.006$ - to $1.087-\mathrm{g} / \mathrm{ml}$ fraction. Division of the apoE between the HDL and $d>1.21-\mathrm{g} / \mathrm{ml}$ fractions was quite variable, the latter having at least 45 and $65 \%$ of the total apoE in the experimental and control perfusates, respectively. These high levels may be, to a large extent, artifactual, being derived primarily from HDL during ultracentrifugation (43).

To overcome this problem, the distribution of the apoE among the perfusate lipoproteins and the $d>1.21$ $\mathrm{g} / \mathrm{ml}$ infranatant fraction was determined after separation by filtration on agarose columns, thereby avoiding ultracentrifugation. Various eluates were pooled according to the peaks illustrated in Fig. 5. The distribution of apoE among the resulting fractions is also shown in the figure. It will be noted that the apoE secreted by the normal liver is approximately equally divided between fractions I (VLDL and LDL), II

\footnotetext{
${ }^{2}$ Dolphin, P. J. Unpublished results.
}

(HDL), and, surprisingly, V. The latter also contained some hemoglobin (because of slight hemolysis during the perfusion), which has a molecular weight of similar magnitude to apoE. However, among lipoproteins secreted by livers from hypercholesterolemic rats, most of the apoE was found' in fraction I with relatively small percentages associated with the lower molecular-weight $(<70,000)$ fractions (III-V).

\section{DISCUSSION}

These data indicate that the rat liver can synthesize and secrete lipoproteins having many of the characteristics of both the circulating B-VLDL of hypercholesterolemic animals (9-12) and the broad-beta lipoproteins of human dysbetalipoproteinemia $(1,44)$. These include VLDL having increased cholesterol ester and decreased triacylglycerol levels and diminished apoC-II and apoC-III content. The VLDL secreted by the fatty livers consistently have slower mobility than the control VLDL on agarose gel electrophoresis, although not sufficiently retarded to show $\beta$-mobility. However, the difference in mobility is similar to that reported by Mjøs et al. (13) after catabolism of triacylglycerol-rich lipoproteins in supradiaphragmatic rats and to the two types of VLDL found in some normolipemic subjects (21).

In addition to cholesterol ester-rich VLDL the liver from hypercholesterolemic rats secretes a cholesterol ester-rich LDL which contains a significant quantity of apoE. However normal livers, in agreement with earlier studies $(42,45)$, secrete virtually no LDL. The secretion of cholesterol ester-rich lipoproteins of $d<1.06 \mathrm{~g} / \mathrm{ml}$ by perfused livers from cholesterol-fed rats has been noted by Swell and Law (46). Indeed, it is possible that the cholesterol-rich VLDL and LDL represent a spectrum of lipoprotein particles, differing only in the relative amounts of lipid and protein. These results do not rule out the possibility that the abnormal LDL is derived from VLDL (or vice versa) within the liver (or in the space of Disse) before its secretion. How-

TABLE VI

Distribution of ApoE among Perfusate Lipoprotein Fractions

\begin{tabular}{lcc}
\hline & Control* $^{*}$ & Hypercholesterolemic \\
\hline & & \% distribution \pm SEM \\
VLDL & $30 \pm 13$ & $17 \pm 3$ \\
LDL & $4 \pm 2$ & $32 \pm 4$ \\
d $>1.087 \S$ & $66 \pm 15$ & $51 \pm 4$ \\
\hline
\end{tabular}

* Three experiments.

\$ Five experiments.

$\S$ The HDL and $d>1.21-\mathrm{g} / \mathrm{ml}$ fractions are not presented separately because of the variable distribution of apoE between them as a result of ultracentrifugal preparation. 
$\%$ DISTRIBUTION OF APO-E

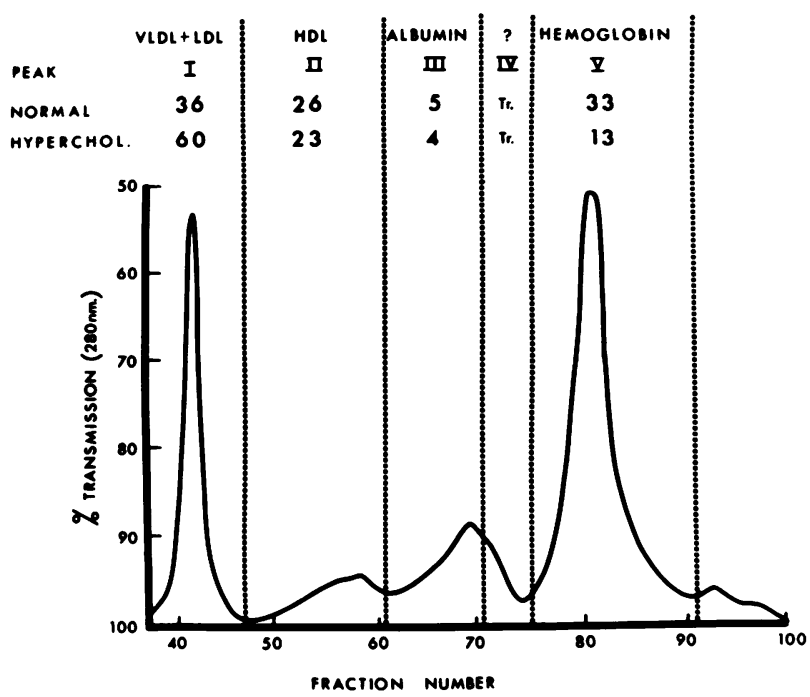

Figure 5 Separation of perfusate lipoproteins by agarose column filtration. The elution pattern illustrated in the figure was obtained with a concentrated perfusate of a liver from a hypercholesterolemic animal. Control liver perfusates gave similar profiles. Approximately $500 \mathrm{mg}$ of protein was applied to each column. The percentage of distribution is the mean of two experiments with each type of liver. The values agreed within $5 \%$. Hyperchol, hypercholesterolemic; Tr, trace.

ever, the formation of abnormal LDL is not a result of the action of the liver during recirculation of the perfusate lipoproteins, because a similar pattern is seen after the use of a nonrecirculating system, nor by lipolysis of normal triacylglycerol-rich VLDL by hepatic lipase.

The distribution of incorporated $\left[{ }^{3} \mathrm{H}\right]$ leucine among the apoproteins of the VLDL and LDL secreted by the livers of hypercholesterolemic rats parallels their pattern on polyacrylamide gel electrophoresis. Thus, at least some of the abnormal cholesterol ester-rich lipoproteins found in the serum of hypercholesterolemic animals are the result of de novo synthesis and not remnants of VLDL or chylomicron catabolism.

The livers secreting the abnormal lipoproteins are infiltrated with lipid, $80 \%$ of which is cholesterol ester. Apparently, the secretion of some of this lipid involves apoE. The association of apoE with cholesterol ester metabolism has been noted in humans (1) and animals (47). The secretion of cholesterol ester-rich lipoproteins and the maintenance of an increased rate of apoE secretion by the perfused fatty livers suggest that the apoprotein plays a role in the transport of cholesterol esters from the liver that is not dependent upon the presence of lipid in the perfusate or, presumably, the serum.

The distribution of apoE among the various density fractions appears to be dependent upon the amount of cholesterol ester to be secreted. Thus in the hypercholesterolemic state, apoE is more firmly bound to the circulating VLDL (14) and less is recovered in the $d>1.21-\mathrm{g} / \mathrm{ml}$ fraction $(12,36)$. In the absence of a cholesterol load, much of the apoE is secreted by the perfused liver as HDL (42), especially when LCAT is inhibited (48). It now appears that the normal liver also secretes a considerable portion of apoE in a virtually delipidated form. The proportion of apoE in fraction V of Fig. $5(<45,000 \mathrm{~mol} \mathrm{wt})$ is greatly diminished in the perfusate of livers from hypercholesterolemic rats (although the absolute quantity is not decreased as much because of the higher secretion rate of the livers from hypercholesterolemic rats), lending credence to the concept that the density fraction in which apoE is found depends upon the amount of cholesterol to be secreted (12). The association of the apoE with the larger cholesterol-carrying lipoproteins may occur in nascent VLDL or in the circulation. Weisgraber et al. (47) noted that after intravenous administration of iodinated HDL, apoE was recovered in HDL of normolipemic rats, but in the B-VLDL, $\mathrm{LDL}$, and $\mathrm{HDL}_{\mathrm{c}}$ of hypercholesterolemic rats. The movement of apoE among lipoprotein fractions may also be reflected by the exchange between VLDL and HDL of an apoprotein having the mobility of apoE on polyacrylamide gel electrophoresis (24).

In addition to redistribution of the $\mathrm{apoE}$ to the cholesterol-carrying lipoproteins, this apoprotein is secreted at a more rapid rate by livers from hypercholesterolemic animals. If it is assumed that the increased rate of synthesis of apoE by the perfused liver is representative of the 6-wk period of the high-fat diet, it can be calculated that $10-15 \mathrm{~d}$ would be required for the level of apoE in sera of hypercholesterolemic rats to reach that found in vivo in $6 \mathrm{wk}$. This suggests that the total catabolic rate is also increased. The potential of the perfused control liver to maintain the normal serum level of $20 \mathrm{mg} / \mathrm{dl}$ (Fig. 1) can be estimated. We have noted an average liver weight of $13 \mathrm{~g}$ for $340-\mathrm{g}$ rats. Assuming a plasma volume of $4.5 \%$ of body weight at the secretion rate of $12 \mu \mathrm{g} / \mathrm{g}$ per $\mathrm{h}$ (Table I) the entire plasma apoE content can be secreted in $20 \mathrm{~h}$. Because the half-life of apoE in the rat has been estimated between 9.5 and $11 \mathrm{~h}(49,50)$, it is apparent that the secretion rate of the perfused normal liver is adequate to maintain the circulating apoE levels. This would be necessary because the intestine secretes virtually no apoE (51).

The origin of the lipoproteins released by the livers from the hypercholesterolemic rats during the first 15 min of perfusion is obscure. Most of it is not a result of lipoproteins in contaminating serum or in extracellular fluid in equilibrium with the circulation, such as in the space of Disse. However, some material 
may be bound to hepatocyte membranes, possibly as remnants, or be released from some damaged hepatocytes. Regardless of the origin of these rapidly released lipoproteins, it is apparent that the fatty livers from the hypercholesterolemic animals are capable of synthesizing de novo and secreting unusual cholesterol ester-rich lipoproteins which have many of the characteristics of the B-VLDL and the LDL seen in vivo, whereas the normal liver secretes the triacylglycerolrich VLDL characteristic of normolipemic serum.

\section{ACKNOWLEDGMENTS}

We wish to thank the Montreal Transfusion Center of the Canadian Red Cross and Mme. M. Caisse for the supply of human blood.

This work was supported by grants MT-1266 and MA-5999 from the Medical Research Council of Canada.

\section{REFERENCES}

1. Shore, B., and V. Shore. 1974. An apolipoprotein preferentially enriched in cholesteryl ester-rich low density lipoprotein. Biochem. Biophys. Res. Commun. 58: 1-7.

2. Havel, R. J., and J. P. Kane. 1973. Primary dysbetalipoproteinemia: predominance of a specific apoprotein species in triglyceride-rich lipoproteins. Proc. Natl. Acad. Sci. U. S. A. 70: 2015-2019.

3. Quarfordt, S. H., R. I. Levy, and D. S. Fredrickson. 1973. The kinetic properties of very low density lipoprotein triglyceride in Type III hyperlipoproteinemia. Biochim. Biophys. Acta. 296: 572-576.

4. Hazzard, W. L., and E. L. Bierman. 1975. Broad- $\beta$ disease versus endogenous hypertriglyceridemia. Levels and lipid composition of chylomicrons and very low density lipoproteins during fat-free feeding and alimentary lipemia. Metab. Clin. Exp. 24: 817-828.

5. Chait, A., J. J. Albers, J. D. Brunzell, and W. R. Hazzard. 1977. Type III hyperlipoproteinemia (remant removal disease). Insight into pathogenetic mechanism. Lancet. I: $1176-1178$.

6. Berman, M., M. Hall, R. I. Levy, S. Eisenberg, D. W. Bilheimer, R. D. Phair, and R. H. Goebel. 1978. Metabolism of apo-B and apo-C lipoproteins in man: kinetic studies in normal and hyperlipoproteinemic subjects. $J$. Lipid Res. 19: 38-56.

7. Mahley, R. W., J. H. Weisgraber, and T. Innerarity. 1974. Canine lipoproteins and atherosclerosis. II. Characterization of the plasma lipoproteins associated with atherogenic and nonatherogenic hyperlipemia. Circ. Res. 35: 722733.

8. Mahley, R. W., K. H. Weisgraber, T. Innerarity, H. B. Brewer, Jr., and G. Assmann. 1975. Swine lipoprotein and atherosclerosis. Changes in the plasma lipoproteins and apoproteins induced by cholesterol feeding. Biochemistry. 14: 2817-2823.

9. Rodriguez, J. L., G. C. Ghiselli, D. Torreggiani, and C. R. Sirtori. 1976. Very low density lipoproteins in normal and cholesterol-fed rabbits: lipid and protein composition and metabolism. Part 1. Chemical composition of VLDL in rabbits. Atherosclerosis. 23: 73-83.

10. Shore, V. G., B. Shore, and R. G. Hart. 1974. Changes in apolipoproteins and properties of rabbit very low density lipoproteins on induction of cholesteremia. Biochemistry. 13: $1579-1585$.
11. Mahley, R. W., K. H. Weisgraber, and T. Innerarity. 1976. Atherogenic hyperlipoproteinemia induced by cholesterol feeding in the Patas monkey. Biochemistry. 15: 29792985.

12. Wong, L., and D. Rubinstein. 1978. The levels of apolipoprotein-E in hypercholesterolemic rat serum. Can. J. Biochem. 56: 161-166.

13. Mjøs, O. D., O. Faergeman, R. L. Hamilton, and R. J. Havel. 1975. Characterization of remnants produced during metabolism of triglyceride-rich lipoproteins of blood plasma and intestinal lymph in rat. J. Clin. Invest. 56: 603-615.

14. Dolphin, P. J., L. Wong, and D. Rubinstein. 1978. A comparison of some immunological characteristics of very low density lipoproteins of normal and hypercholesterolemic rat sera. Can. J. Biochem. 56: 673-683.

15. Sigurdson, G., S-P. Noel, and R. J. Havel. 1978. Catabolism of the apoprotein of low density lipoproteins by the isolated perfused rat liver. J. Lipid Res. 19: 628-634.

16. Miller, L. L., C. G. Bly, M. L. Watson, and W. F. Bale. 1951. The dominant role of the liver in plasma protein synthesis. A direct study of the isolated perfused rat liver with the aid of lysine $\epsilon-C^{14}$ J. Exp. Med. 94: 431-453.

17. Hamilton, R. L., M. N. Berry, M. C. Williams, and E. M. Severinghaus. 1974. A simple and inexpensive membrane "lung" for small organ perfusion. J. Lipid Res. 15: $182-186$.

18. Havel, R. J., H. A. Eder, and J. H. Bragdon. 1955. The distribution and chemical composition of ultracentrifugally separated lipoproteins in human serum. J. Clin. Invest. 34: 1345-1353.

19. Radding, C. M., and D. Steinberg. 1960. Studies on the synthesis and secretion of serum lipoproteins by rat liver slices. J. Clin. Invest. 39: 1560-1569.

20. Noble, R. R. 1968. Electrophoretic separation of plasma lipoproteins in agarose gel. J. Lipid Res. 9: 693-700.

21. Pagnan, A., R. J. Havel, J. P. Kane, and L. Kotite. 1977. Characterization of human very low density lipoproteins containing two electrophoretic populations: double pre-beta lipoproteins and primary dysbetalipoproteinemia. J. Lipid Res. 18: 613-622.

22. Sata, T., D. L. Estrich, P. D. S. Wood, and L. W. Kinsel. 1970. Evaluation of gel chromatography for plasma lipoproteins. J. Lipid Res. 11: 331-340.

23. Scanu, A. M., and C. Edelstein. 1971. Solubility in aqueous solutions of ethanol of the small molecular weight peptides of the serum VLDL and HDL. Anal. Biochem. 44: 576-588.

24. Rubenstein, B., and D. Rubinstein. 1972. Interrelationship between rat serum very low density and high density lipoproteins. J. Lipid Res. 13: 317-324.

25. Dolphin, P. J., and D. Rubinstein. 1977. Glycosylation of apoproteins of rat very low density lipoproteins during transit through the hepatic Golgi apparatus. Can.J. Biochem. 55: 83-90.

26. Laurell, C-B. 1965. Quantitative estimation of proteins by electrophoresis in agarose gel containing antibodies. Anal. Biochem. 15: 45-52.

27. Bradford, M. M. 1976. A rapid and sensitive method for the quantitation of microgram quantities of protein utilizing the principle of protein-dye binding. Anal. Biochem. 72: 248-254.

28. Folch, J., M. Lees, and G. H. Sloane Stanley. 1957. A simple method for the isolation and purification of total lipids from animal tissues. J. Biol. Chem. 226: 497-509.

29. Fiske, C. H., and Y. SubbaRow. 1926. The colorimetric determination of phosphorous. J. Biol. Chem. 66: 375400. 
30. Van Handel, C. 1961. Suggested modifications of the micro determination of triglycerides. Clin. Chem. 7: 249251.

31. Zlatkis, A., B. Zak, and A. J. Boyle. 1953. A new method for the direct determination of serum cholesterol. $J$. Lab. Clin. Med. 41: 486-492.

32. Nilsson-Ehle, P., and M. C. Scholtz. 1976. A stable radioactive substrate emulsion for assay of lipoprotein lipase. J. Lipid Res. 17: 536-541.

33. Lowry, O. H., N. J. Rosebrough, A. L. Farr, and R. J. Randall. 1951. Protein measurement with the Folin phenol reagent. J. Biol. Chem. 193: 265-275.

34. Schmidt, G., and S. J. Thannhauser. 1945. A method for the determination of desoxyribonucleic acid, ribonucleic acid, and phosphoproteins in animal tissues. J. Biol. Chem. 161: 83-89.

35. Schneider, W. C. 1945. Phosphorus compounds in animal tissues. I. Extraction and estimation of deoxypentose nucleic acid. J. Biol. Chem. 161: 293-303.

36. Mahley, R. W. and K. S. Holcombe. 1977. Alterations of the plasma lipoproteins and apoproteins following cholesterol feeding in the rat. J. Lipid Res. 18: 314-323.

37. Felker, T. E., M. Fainaru, R. L. Hamilton, and R. J. Havel. 1977. Secretion of the arginine rich and A-1 apolipoproteins by the isolated perfused rat liver. J. Lipid Res. 18: 465-473.

38. Seidel, D., H. V. Buff, V. Faucer, and U. Bleyl. 1976. On the metabolism of lipoprotein X (Lp-X). Clin. Chim. Acta. 66: 195-207.

39. Koen, A. L., and C. R. Shaw. 1964. A preparative method employing starch gel electrophoresis and electrodialysis. Anal. Biochem. 9: 495-498.

40. Fainaru, M., R. J. Havel, and K. Imaizumi. 1977. Radioimmunoassay of arginine-rich apolipoproteins of rat serum. Biochim. Biophys. Acta. 490: 144-155.

41. Marsh, J. B. 1974. Lipoproteins in a non-recirculating perfusate of rat liver. J. Lipid Res. 15: 544-550.
42. Marsh, J. B. 1976. Apoproteins of the lipoproteins in a non-recirculating perfusate of rat liver. J. Lipid Res. 17: 85-90.

43. Fainaru, M., R. J. Havel, and D. Imaizumi. 1977. Apoprotein content of plasma lipoproteins of the rat separated by gel chromatography. Biochem. Med. 17: 247-255.

44. Hazzard, W. R., D. Porte, Jr., and E. L. Bierman. 1972. Abnormal lipid composition of very low density lipoproteins in diagnosis of broad-beta disease (Type III hyperliproteinemia). Metab. Clin. Exp. 21: 1009-1019.

45. Noel, S-P., and D. Rubinstein. 1974. Secretion of apolipoprotiens in very low density and high density lipoprotein by perfused rat liver. J. Lipid Res. 15: 301-308.

46. Swell, L., and M. D. Law. 1971. Release of lipoprotein cholesterol esters by the isolated perfused rat liver. Biochim. Biophys. Acta. 231: 302-313.

47. Weisgraber, K. H., R. W. Mahley, and G. Assmann. 1977. The rat arginine-rich apoprotein and its redistribution following injection of iodinated lipoproteins into normal and hypercholesterolemic rats. Atherosclerosis. 28: 121140.

48. Hamilton, R. L., M. C. Williams, C. J. Fielding, and R. J. Havel. 1976. Discoidal bilayer structure of nascent high density lipoproteins from perfused rat liver. J. Clin. Invest. 58: 667-680.

49. Eisenberg, S., H. G. Windmueller, and R. I. Levy. 1973. Metabolic fate of rat and human lipoprotein apoproteins. J. Lipid Res. 14: 446-454.

50. Roheim, P. S., D. Rachmilewitz, O. Stein, and Y. Stein. 1971. Metabolism of iodinated high density lipoproteins in the rat. I. Half-life in the circulation and uptake by organs. Biochim. Biophys. Acta. 248: 315-329.

51. Wu, A-L., and H. G. Windmueller. 1978. Identification of circulating apolipoproteins synthesized by rat small intestine in vivo. J. Biol. Chem. 253: 2525-2528. 